\title{
Fusion of Medical Images Using Adaptive Pulse Coupled Neural Networks Based on OCSA Optimization
}

\author{
Pydi Kavita1, Daisy Rani Alli² and Annepu Bhujanga Rao ${ }^{2}$ \\ ${ }^{1}$ Department of Instrument Technology, Andhra University, AP, India \\ ${ }^{2}$ Department of Instrument Technology, Andhra University, Visakhapatnam, AP, India \\ Corresponding author email: kavitaspydi@gmail.com
}

\begin{abstract}
The goal for fusion of image is to incorporate descriptions of the same scene from multiple images. A new image that is more appropriate for human and machine interpretation or more image-processing tasks such as segmentation, extraction of features and object detection is the result of image fusion. In this article, fusion of MRI and CT images are proposed and new model is designed to approach the fusion based on neural networks and Optimization technique which works better and gives good results. In this a paper, an adaptive pulse coupled neural networks (PCNN) is used to determine the right parameters, these parameters are optimized using Quantum Cuckoo Search Algorithm (QCSA). The reliability and accuracy of the image is increased by performing optimization technique. The fitness function of the proposed optimization technique is defined using image entropy (EN), average gradient (AG) and spatial frequency (SF) of image for finding the optimal solution. Various parametric values are being tested to show that the suggested QCSA-PCNN is superior compared with other current technique like QPSOPCNN. The PSNR obtained using QPSO-PCNN is 40.82, the proposed QCSA-PCNN the Peak signal to noise ratio (PSNR) value is 43.79. The QCSA-PCNN method has good Structural Similarity Index (SSIM) with 0.99 compared to QPSO which has 0.96. The experimental results are conducted using MAT Lab method.
\end{abstract}

KEY WORDS: IMAGE FUSION, PULSE COUPLED NEURAL NETWORKS, FITNESS FUNCTION, QPSO, QCSA.

\section{INTRODUCTION}

Image fusion is a method for using image processing techniques to merge multimodal images. Specifically, the goal is to combine diverse and complementary evidence in order to boost the details visible in the photographs and to maximize the clarity of the interpretation (ShihGu Huang 2010, S.Vasuki et al., 2012). Complementary input from multiple modalities is required because of the introduction of new diseases. For scanning of sensitive tissues, such as the brain technology used are MRI and CT scans which are helpful in obtaining the depth of

Biosc Biotech Res Comm P-ISSN: 0974-6455 E-ISSN: 2321-4007

\section{crossef}

Identifiers and Pagination

Year: 2021 Vol: 14 No (5) Special Issue

Pages: 182-189

This is an open access article under Creative

Commons License Attribn 4.0 Intl (CC-BY).

DOI: http://dx.doi.org/10.21786/bbrc/14.5/33 problem involved. The best information on denser tissue is provided by CT and better soft tissue information is provided by MRI (G. Mamatha et al., 2012). These complementarities have contributed to the belief that integrating photographs obtained with multiple medical instruments would yield an image that can contain more data than individual images. The accuracy of result can be high by merging of two images i.e. MRI image and CT image (A.S Sekhar et al., 2011). The fusion of images is closely connected to many various areas of image processing, such as Images acquired form satellite, dense areas, and highly recommended in medical field. Initially the research on image fusion is performed for satellite imaging, based on the results obtained the field of research is extended for medical imaging.

With each fusion process, different methods are classified into a spatial domain (such as Principal Component Analysis (PCA), Hue Strength Saturation (HIS) and Brovey Transform) and a transform domain (such as Discrete Wavelet Transform (DWT), Curvelet Transform (CT), 
Non-sampled Contoured Transform (NSCT)) (Shavetha M et al., 2014 \&t A Kaur et al.,2016). The multi-resolution single value decomposition (MSVD) was introduced by R Kakarala et al., (2001) from the principle of singular value decomposition.

The MSVD transformation allows it possible to decompose the picture into sub-bands of low pass and high pass. MSVD is used and thanks to the multiresolution property for various multi-resolution signal and image manipulation, such as image fusion ( $S$ Goutam et al., 2015 \& M Varsha et al., 2014) and image de-noising (S Malini et al., 2015). The MSVD dependent picture fusion method is suggested in (V.P.S Naidu, 2011) this gives a reliable finding that is better than wavelets. It is very simple to implement, very easy to quantify, and has no defined range of fundamental vectors, such as the wavelet and the Discrete Cosine transformation (DCT). The Pulse-coupled Neural Network (PCNN) is a biologically guided neural network. It has the global binding and pulse synchronous character of neurons. In recent literature, several image fusion algorithms based on the PCNN model have been created in several works that provide good results (M Subashini et al., 2014).

Intelligent optimization algorithms have been implemented by several researchers to improve PCNN parameters, and many adaptive PCNN models have been suggested, such as PCNN (Z Wang et al., 2015), PSO-PCNN (X Z Zu et al., 2011), QPSO-PCNN (S F Ding et al., 2012), MDE-PCNN (X.Y Ziang et al., 2012), and FOA-PCNN (H Zhoa et al, 2014). The above processes, though, have their own shortcomings and need to be solved. For example, to enhance the search effectiveness and generalization ability, the fitness function of these optimization algorithms should be changed. In this article, the algorithm for Quantum Cuckoo search optimization is considered and assessed. The presence of cuckoo quest has two phases: one is the development process and the other is the refining phase in which it is possible to achieve the optimal solution.

Figure 1: Structure of PCNN neuron

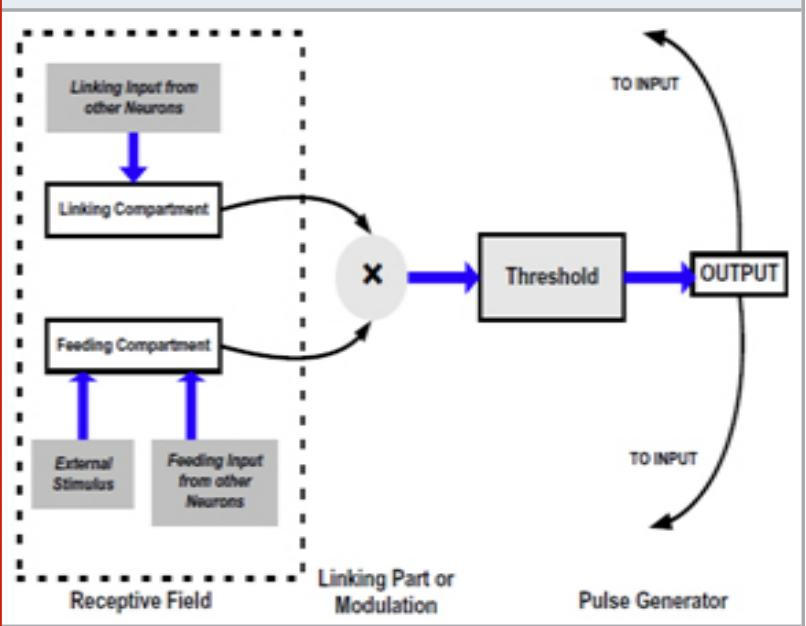

\section{MATERIAL AND METHODS}

PCNN Models: One of the common models which are considered for various image processing applications are PCNN. Some of the applications are segmentation of image, de-noising of image, attribute detection, recognition of pattern, fusion of images etc. (M Subashini et al., 2014) The PCNN model consists of two points of distinction. The first one is that neurons tend to pulse together with similar intensities correlated with each community of spatially linked pixels. Secondly, depending on the value of threshold, this decreases the other neurons exponentially. There are three sections to the PCNN model: input receptive field, connecting modulation and generator of pulse, as seen in Fig. 1 of Z.Wang et al., 2015.

The receptive area is the portion of the input that consists of the linking and the feeding networks, two forms of inputs. The distinction among the inputs lies in the truth that external stimuli are provided to the linking connections. Moreover, they have a continuous reaction time that is higher than the feeding ties that receive external and local stimuli. The following equations given below describe the model of PCNN for $n^{\text {th }}$ iteration:

$$
\begin{aligned}
& F_{i j}[n]=e^{\left(\alpha_{F} \delta_{n}\right)} F_{i j}[n-1]+S_{i j}+V_{F} \sum_{k l} M_{i j k l} Y_{k l}[n-1] \\
& L_{i j}[n]=e^{\left(\alpha_{L} \delta_{n}\right)} L_{i j}[n-1]+V_{L} \sum_{k l} W_{i j k l} Y_{k l}[n-1]
\end{aligned}
$$

$\mathrm{F}_{\mathrm{ij}}[\mathrm{n}]$ is said to be the initial feeding compartment of the neurons $\mathrm{i}$ and $\mathrm{j} . \mathrm{L}_{\mathrm{ij}}$ [n]Is the output of the compartment linking $\mathrm{S}_{\mathrm{ij}}$ is the stimulus of input image pixels in positions of $\mathrm{i}$ and $\mathrm{j}$. $\mathrm{Y}_{\mathrm{kl}}$ Indicate the neurons output based on the previous iteration [n -1];

$\mathrm{M}_{\mathrm{ijkl}}$ and $\mathrm{W}_{\mathrm{ijkl}}$ are the Gaussian weight functions that are constant with the distance;

The voltage i.e. inherent of the network is termed as $V_{F}$ and $\mathrm{V}_{\mathrm{L}}$

The internal state of neurons are created by combining the states of two compartments and the activity of neuron $\mathrm{U}_{\mathrm{ij}}[\mathrm{n}]$ is evaluated using equation (3) which is given below,

$\mathrm{U}_{\mathrm{ij}}[\mathrm{n}]=\mathrm{F}_{\mathrm{ij}}[\mathrm{n}]\left(1+\beta_{\mathrm{ij}} \mathrm{L}_{\mathrm{ij}}[\mathrm{n}]\right)$

$\beta$ is the coefficient of linking

To produce pulsating output ' $Y$ ' the internal state of the neuron is compared to a threshold $\theta_{\mathrm{ij}}$

$$
Y_{i j}[n]=\left\{\begin{array}{c}
1, U_{i j}[n]>\theta_{i j}[n] \\
0, \text { Otherwise }
\end{array}\right.
$$

When the pulsating output is greater than the threshold value, the neurons will improve, which directly leads to 
increase in threshold value. The threshold value gradually decreases until the next neuron gets fired. In this process the threshold value is said to be dynamic. The process is described by,

$$
\theta_{i j}[n]=e^{\left(-\alpha_{T}\right)} \theta_{i j}[n-1]+V_{\theta} Y_{i j}[n-1]
$$

Where $\mathrm{V}_{\theta}$ is represented as a large constant greater than the average value of $\mathrm{U}_{\mathrm{ij}}$.

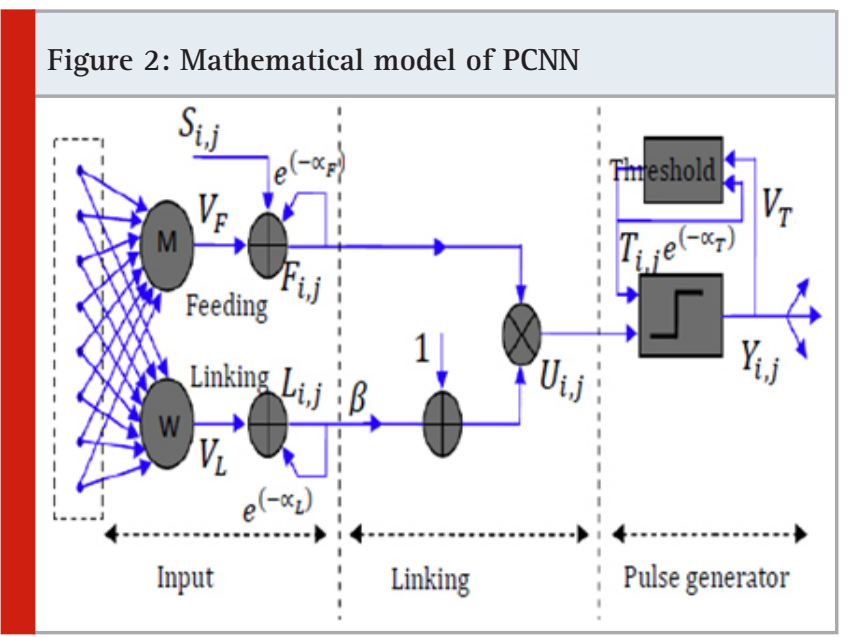

Quantum Cuckoo Search Algorithm: In standard cuckoo search (CS) all the cuckoos have indistinguishable pursuit practices. Thus, all the people of the populace have a similar system to compute the new update. It depends on the worldwide best arrangement and the current one. Along these lines, the calculation may trap in neighborhood optima prompting untimely assembly. Anyway in quantum cuckoo search (QCS) non homogeneous update is performed by choosing arbitrarily one update of three ones with a similar likelihood of appearance. The first is the update given in standard CS. While, the two different ones are motivated from the quantum hypothesis.

The quantum hypothesis has acquainted new ideas with old style material science. In any case, the most principal condition of the quantum hypothesis is the Schrödinger condition, created in 1926 by the Austrian physicist Erwin Schrödinger. It has significant applications in material science and different fields. The condition is a sort of differential condition known as a wave condition, which fills in as a numerical model of the development of waves. The time-subordinate Schrödinger wave condition for a particle decides how the wave capacities develop through reality and it is represented by the following condition:

$$
j h \frac{\partial}{\partial t} \Psi(r, t)=\left[\frac{h^{2}}{2 m} \nabla^{2}+V(r, t)\right] \Psi(r, t)
$$

where, $\mathrm{j}$ is imaginary unit, $\mathrm{h}$ is the reduced Planck constant, $\Psi(\mathrm{r}, \mathrm{t})$ is the wave function of the quantum system at position and time, is particle's mass, ( ) is the potential and is the Laplacian operator. In QCS, each cuckoo is viewed as a particle traveling in an $\mathrm{N}$-dimensional Hilbert space with a given energy, and in this way its condition is described by a wave work that relies solely on the circumstance of the molecule. In the long run, the condition of the molecule is calculated using the Monte Carlo backwards shift. The way to approximate the condition of the molecule in quantum mechanics is solved by falling the quantum state to the conventional state. The solution of $|\mathrm{X}|$ isgiven by:

$$
|X|=\frac{L}{2} \ln \left(\frac{1}{\eta}\right)
$$

A random number uniformly distributed on $(0,1)$ is $\eta$ and $\ln$ is the natural logarithmfunction.

By putting $L=2 \delta\left|\bar{x}-x_{i}\right| \ln \left(\frac{1}{\eta}\right)$

The current position $\mathrm{x}$ _iand the mean position $\mathrm{x} \mathrm{x}^{-}$of the population of size $n$ is calculated by:

$$
\bar{x}=\sum_{j=1}^{n} x_{j}
$$

The first new update is given by the following equation:

$$
x_{i+1}=\bar{x}+\delta *\left(\bar{x}-x_{i}\right) * \ln (1 / r)
$$

Where $\delta$ is a control parameter, and $r$ is a random number taken from a uniform distribution interval of [0, 1]. In order to ensure that there is no significant fluctuation across the world's best nest $\left(\mathrm{x}_{\mathrm{b}}\right)$, the second latest upgrade is based on the equation (10) given below,

$$
x_{i+1}=\bar{x}+\delta *\left(x_{b}-x_{i}\right) * \exp (r)
$$

Proposed PCNN-QCSA Image Fusion: Three parameters $\alpha \theta, \beta$ and $\mathrm{V} \theta$ are important for obtaining the results of image fusion in PCNN model. The figure 3 shows the process of proposed methodology in which the optimization technique i.e. QCSA which is used to optimize the PCNN model to achieve optimal solution for the parameters evolved in PCNN. The Cuckoo is initialised for defining the spatial solution $\mathrm{x}(\alpha \theta, \beta$ and $\mathrm{V} \theta)$ and the best cuckoo need to identify which is given as $x_{b}$ ( $\alpha \theta, \beta$, and $\mathrm{V} \theta$ ) for configuring the best optimal solution of parameters in PCNN.

Possible parameter configuration schemes, which establish an answer space for boundary improvement are depicted in our proposed interaction. Cuckoo in the population is partitioned into $\mathrm{m}$ gatherings in the wake of delivering an underlying cuckoo arrangement space, and each gathering is subject to each other. Beginning with the underlying arrangement, the cuckoo initially plays out an intra-class enhancement through a neighbourhood search in every classification, continually refreshing their own wellness esteems. The proficiency of the entire cuckoo populace is advanced in $\mathrm{N}$ emphases of neighbourhood improvement with the upgrade of the nature of cuckoos on the whole classes. 
In consistence with the characterized law, the cuckoos of the population are then intertwined and pulled together and neighbourhood advancement inside the gathering is done until the last states of emphasis are met. Finally, the global optimal cuckoo population solution is defined as the optimal configuration of the PCNN parameters. Using the optimum parameter setup above, the final fusion picture is thus obtained.

Figure 3. Proposed model of image fusion i.e. PCNNQCSA

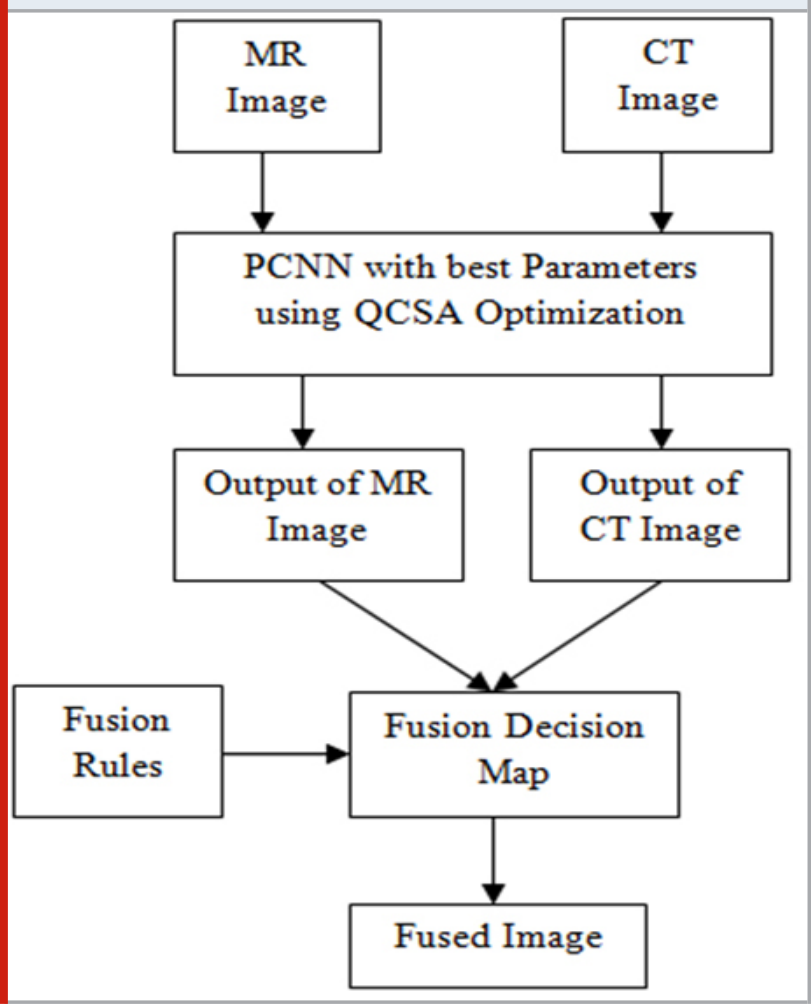

\section{RESULTS AND DISCUSSION}

Fusion performance using QPSO and QCSA is obtained and separate parametric values are also determined, taking into account various combinations of CT image and MR image. In this experiment, picture sharpness, reciprocal knowledge, standard deviation (STD), spatial frequency (SF) are used as the standard for estimating the output of various methods for the purpose of objectively judging different methods. Structural similarity (SSIM), image entropy and Mutual Information (MI) have also been used to approximate the efficiency of various methods. The amount of knowledge that the picture holds is defined by image entropy. The standard deviation reflects the pixel gray level distribution of the image. The smaller the standard deviation is, the greater the comparison. The level of similarity between the groundtruth image and output image is defined as Structural similarity (SSIM) was proposed by Z. Wang et al.,(2004). The results are performed on various set of images.

Image2: In this condition we consider different image to test the level of accuracy of the obtained results. The more the test cases the better the illustration of proposed methodology.

Figure 4: Process Flowchart of PCNN optimization using QCSA

Initialise the essential parameters $\alpha_{8}, \beta$ and $V_{8}$

Produce initial solution $x\left(a_{8}, \beta\right.$ and $\left.V_{8}\right)$

Produce $i^{\text {th }}$ cuckoo population
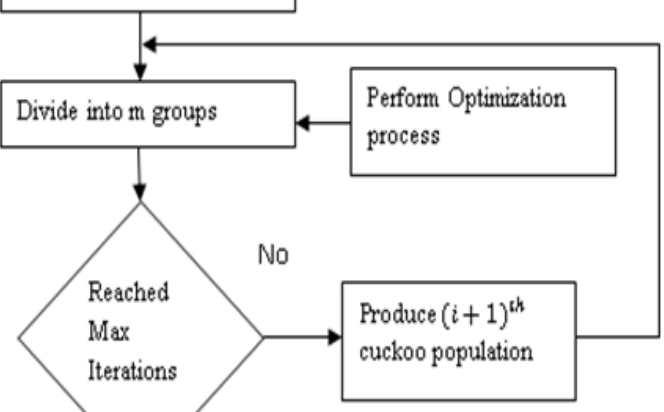

Global Optimal Solution

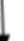

Output the Optimal results of PCNN parameters

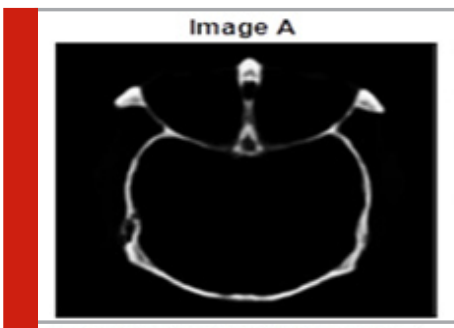

Fused Image using QPSO
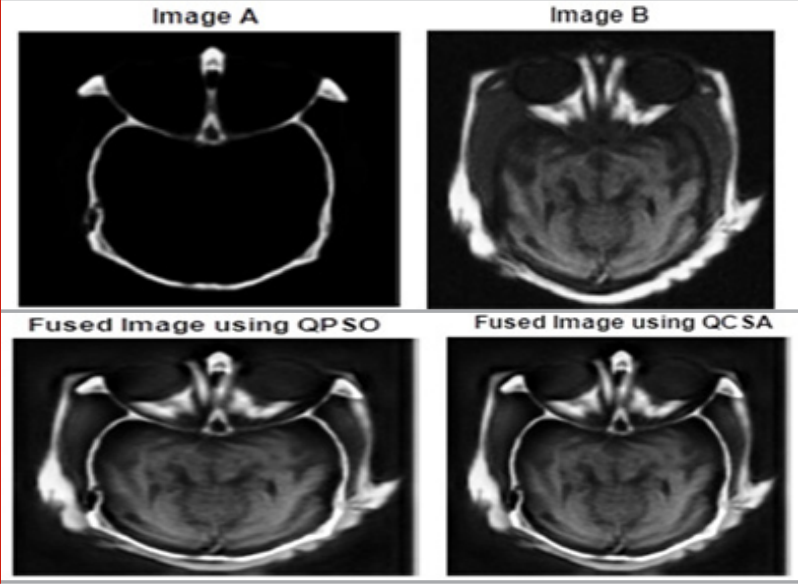

Fused Image using QC SA

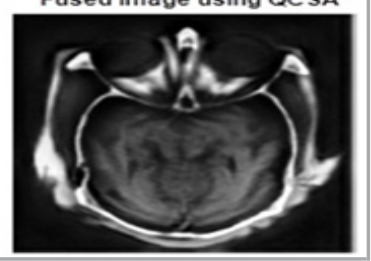

The overall performance of the proposed methodology is shown in terms of PSNR (Peak Signal to Noise Ratio) and SSIM (Structural Similarity Index). These parametric values are evaluated using QPSO-PCNN and QCSA-PCCN methods. The proposed method shows good results in terms of both PSNR and SSIM as shown in table 5. 
Table 1. Parametric Values evaluated for Image1

\begin{tabular}{|c|c|c|c|c|c|c|c|c|c|}
\hline $\begin{array}{l}\text { Parameters/ } \\
\text { Technique }\end{array}$ & Sharpness & STD & Entropy & $\begin{array}{c}\text { Spatial } \\
\text { Freq }\end{array}$ & SSIM A & SSIM B & MI_A & MI_B & MI_F \\
\hline Proposed QCSA & 14.55 & 57.3 & 7.09 & 89.8 & 0.68 & 0.74 & 7.08 & 6.73 & 2.09 \\
\hline QPSO & 7.76 & 65.1 & 6.34 & 23.5 & 0.75 & 0.70 & 3.79 & 3.28 & 0.58 \\
\hline $\begin{array}{l}\text { Laplacian } \\
\text { Approach } \\
\text { (P J Burt et al., 1983) }\end{array}$ & 7.083 & 56.99 & 4.52 & 19.63 & 0.75 & 0.66 & 0.95 & 0.90 & 1.86 \\
\hline Dual channel PCNN (Y.D Ma et al., 2008) & 1.025 & 15.35 & 3.96 & 6.63 & 0.66 & 0.671 & 0.89 & 0.84 & 1.73 \\
\hline MDE PCNN (G Y Wang et al., 2015) & 3.87 & 34.85 & 4.53 & 10.26 & 0.75 & 0.674 & 1.71 & 1.00 & 2.72 \\
\hline EL-DC-PCNN (Y Li et al., 2014) & 3.89 & 35.65 & 4.53 & 9.99 & 0.75 & 0.670 & 1.72 & 1.00 & 2.72 \\
\hline
\end{tabular}

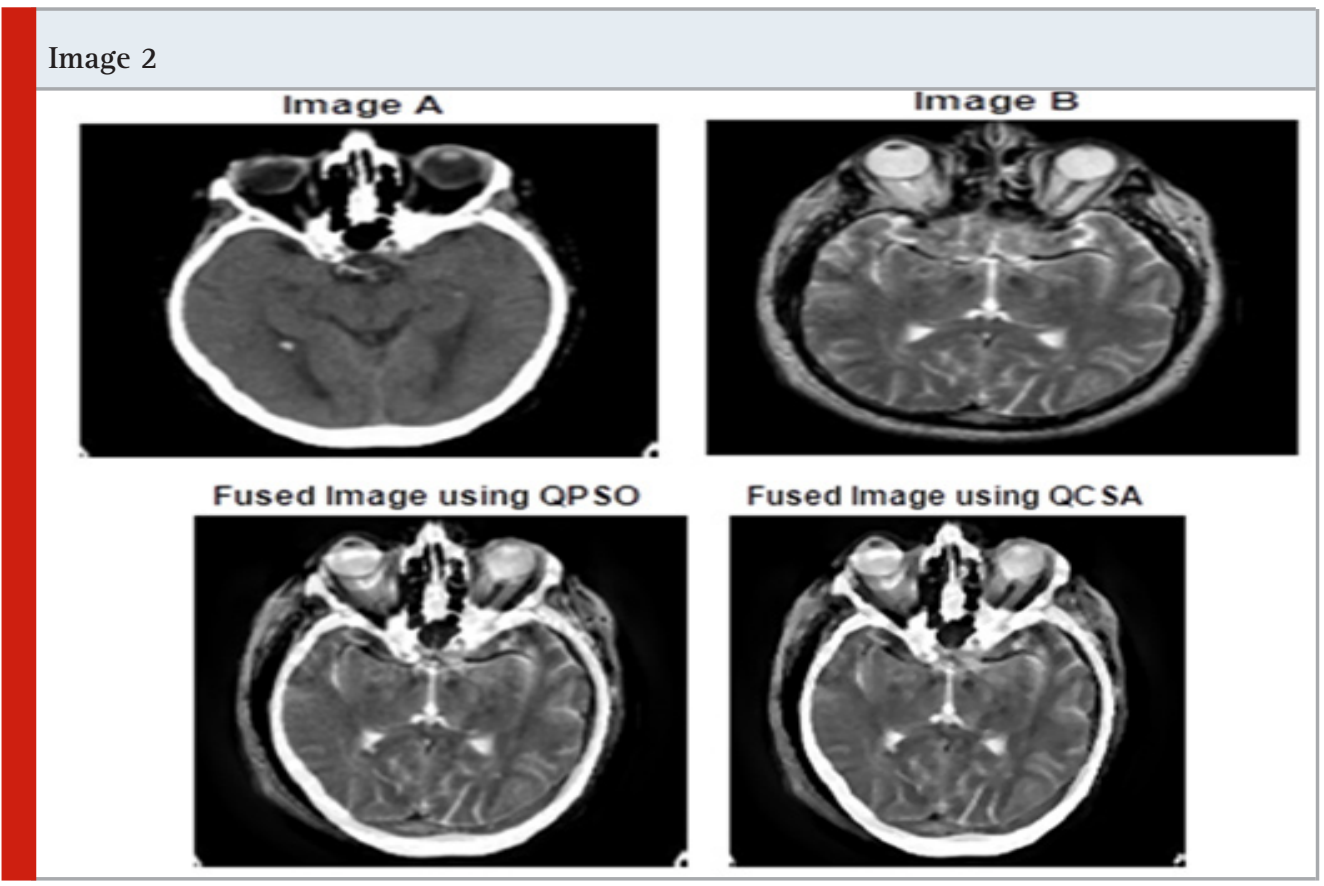

Table2. Parametric Values evaluated for Image2

\begin{tabular}{|c|c|c|c|c|c|c|c|c|c|}
\hline $\begin{array}{l}\text { Parameters/ } \\
\text { Technique }\end{array}$ & Sharpness & STD & Entropy & $\begin{array}{c}\text { Spatial } \\
\text { Freq }\end{array}$ & SSIM A & SSIM B & MI_A & MI_B & MI_F \\
\hline Proposed QCSA & 15.8 & 76.5 & 6.33 & 62.9 & 61.5 & 0.64 & 6.34 & 5.68 & 4.86 \\
\hline QPSO & 8.2 & 60.35 & 4.65 & 25.2 & 76.7 & 0.67 & 2.77 & 1.77 & 1.09 \\
\hline $\begin{array}{l}\text { Laplacian Approach } \\
\text { (P J Burt et al., 1983) }\end{array}$ & 8.124 & 57.67 & 5.18 & 20.14 & 0.78 & 0.55 & 1.04 & 0.68 & 1.73 \\
\hline Dual channel PCNN (Y.D Ma et al., 2008) & 1.082 & 16.28 & 4.15 & 7.23 & 0.514 & 0.85 & 0.87 & 1.03 & 1.90 \\
\hline MDE PCNN (G Y Wang et al., 2015) & 4.43 & 35.65 & 5.28 & 10.80 & 0.749 & 0.67 & 1.20 & 1.38 & 2.58 \\
\hline EL-DC-PCNN (Y Li et al., 2014) & 4.88 & 36.34 & 5.35 & 10.87 & 0.795 & 0.56 & 1.89 & 1.03 & 2.93 \\
\hline
\end{tabular}


Kavita et al.,

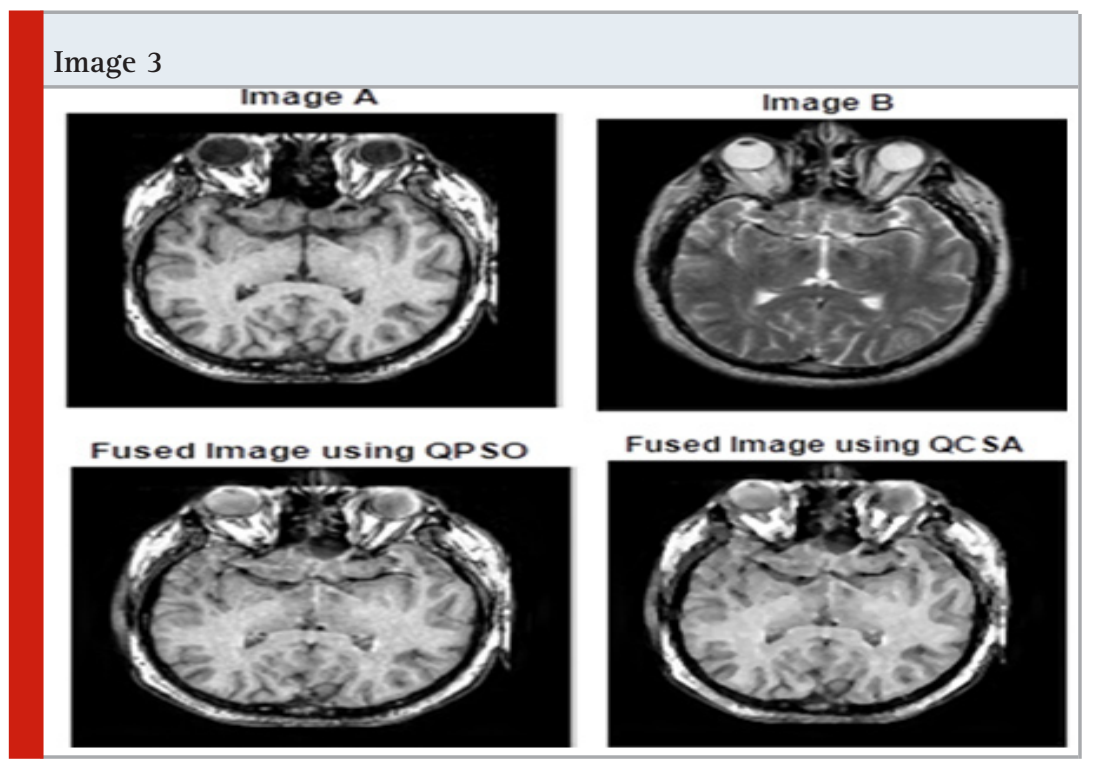

Table 3. Parametric Values evaluated for image3

\begin{tabular}{|c|c|c|c|c|c|c|c|c|c|}
\hline $\begin{array}{l}\text { Parameters/ } \\
\text { Technique }\end{array}$ & Sharpness & STD & Entropy & $\begin{array}{c}\text { Spatial } \\
\text { Freq }\end{array}$ & SSIM A & SSIM B & MI_A & MI_B & MI_F \\
\hline Proposed QCSA & 19.04 & 81.3 & 6.18 & 105.4 & 0.82 & 0.99 & 6.18 & 5.57 & 5.81 \\
\hline QPSO & 7.12 & 68 & 5.66 & 22.8 & 0.79 & 0.58 & 3.23 & 2.15 & 1.15 \\
\hline $\begin{array}{l}\text { Laplacian Approach } \\
\text { (P J Burt et al., 1983) }\end{array}$ & 8.21 & 57.67 & 5.18 & 20.16 & 0.78 & 0.55 & 1.04 & 0.688 & 1.738 \\
\hline Dual channel PCNN (Y.D Ma et al., 2008) & 1.102 & 16.28 & 4.15 & 7.23 & 0.51 & 0.85 & 0.87 & 1.035 & 1.908 \\
\hline MDE PCNN (G Y Wang et al., 2015) & 4.63 & 35.93 & 5.41 & 11.80 & 0.76 & 0.566 & 2.08 & 1.055 & 3.143 \\
\hline EL-DC-PCNN (Y Li et al., 2014) & 4.96 & 36.42 & 5.42 & 11.03 & 0.78 & 0.56 & 2.11 & 1.065 & 3.15 \\
\hline
\end{tabular}
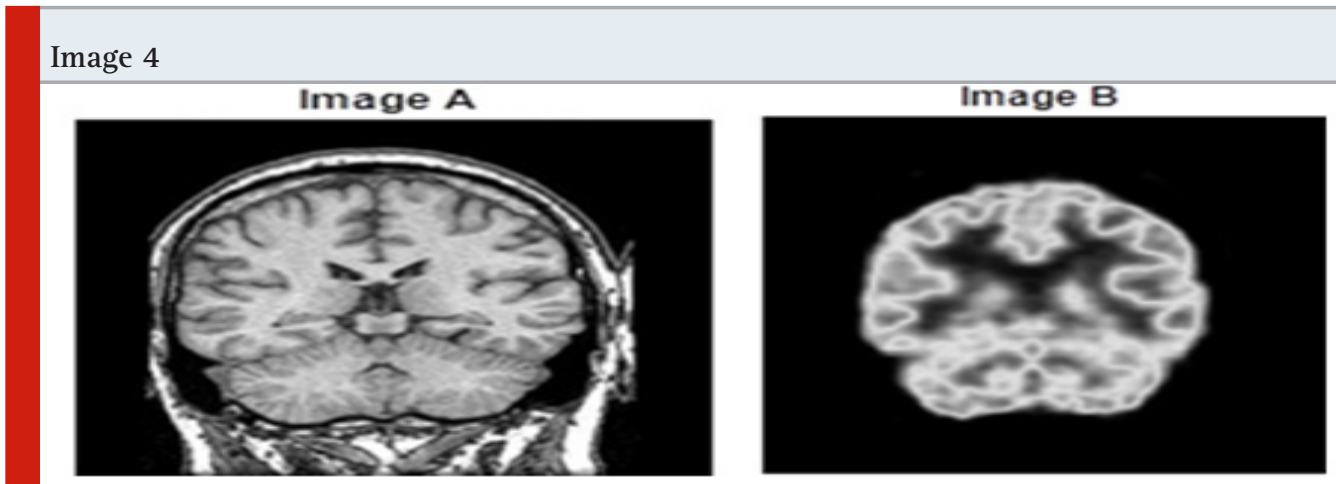

Fused Image using OPSO

Fused Image using QC SA
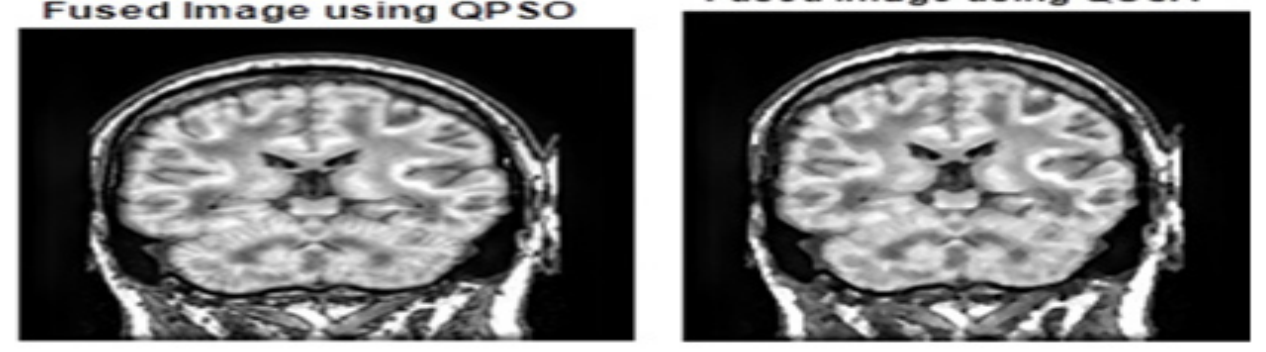
Table 4. Parametric Values evaluated for Image4

\begin{tabular}{|c|c|c|c|c|c|c|c|c|c|}
\hline $\begin{array}{l}\text { Parameters/ } \\
\text { Technique }\end{array}$ & Sharpness & STD & Entropy & $\begin{array}{l}\text { Spatial } \\
\text { Freq }\end{array}$ & SSIM A & SSIM B & MI_A & MI_B & MI_F \\
\hline Proposed QCSA & 20.8 & 84.7 & 19.6 & 19.6 & 0.76 & 0.99 & 6.7 & 3.9 & 6.1 \\
\hline QPSO & 5.55 & 63.3 & 25.88 & 25.8 & 0.79 & 0.59 & 3.6 & 2.4 & 1.3 \\
\hline $\begin{array}{l}\text { Laplacian Approach } \\
\text { (P J Burt et al., 1983) }\end{array}$ & 6.123 & 55.6 & 3.56 & 19.87 & 0.938 & 0.66 & 0.99 & 0.65 & 1.64 \\
\hline Dual channel PCNN (Y.D Ma et al., 2008) & 1.968 & 14.7 & 3.18 & 6.84 & 0.746 & 0.763 & 0.84 & 0.85 & 1.69 \\
\hline MDE PCNN (G Y Wang et al., 2015) & 3.30 & 33.6 & 3.72 & 11.10 & 0.89 & 0.737 & 1.276 & 1.046 & 2.32 \\
\hline EL-DC-PCNN (Y Li et al., 2014) & 3.35 & 34.7 & 3.9 & 9.48 & 0.87 & 0.735 & 1.277 & 1.10 & 2.37 \\
\hline
\end{tabular}

Table 5. Comparison of PSNR and SSIM

\begin{tabular}{|l|l|c|c|c|}
\multirow{2}{*}{$\begin{array}{l}\text { Different images/ } \\
\text { Parameters }\end{array}$} & \multicolumn{2}{|c|}{ PSNR } & \multicolumn{2}{c|}{ SSIM } \\
\cline { 2 - 5 } & QPSO & QCSA & QPSO & QCSA \\
\hline Image 1 & 40.82 & 43.79 & 0.96 & 0.99 \\
\hline Image 2 & 41.37 & 42.30 & 0.96 & 0.99 \\
\hline Image 3 & 40.67 & 40.37 & 0.96 & 0.99 \\
\hline Image 4 & 41.9 & 44.9 & 0.95 & 0.99 \\
\hline
\end{tabular}

\section{CONCLUSION}

To improve the human visual perception the images need to be enhanced. One of the promising techniques is image fusion method, which helps in producing a new enhanced image. The image fusion was carried over for multimodal images obtained from CT and MRI scans. One of the most effective techniques for fusion of medical images is PCNN. To obtain quantitative analysis of image fusion effects important assessment parameters need to be considered. The parameters are image entropy (EN), average gradient (AG) and spatial frequency (SF). The comparative study of image fusion techniques helps in selecting the best fusion approach and hence PCNNQCSA achieves better visual concept of the fused image. The parameters tested show that PCNN-QCSA is strong relative to PCNN-QPSO. The final fusion performance is proved to be best in terms of PSNR and SSIM. The proposed PCNN-QCSA gives 99\% SSIM and 44.9 PSNR value, this leads the proposed methodology to be used in wide range of medical applications.

\section{ACKNOWLEDGMENTS}

I would like to thank department of Instrument Technology, AU College of Engineering for providing excellent research facilities for successful completion of my publication.

Conflict of Interest: No conflict of Interest

\section{REFERENCES}

A. S. Sekhar and M. N. G. Prasad (2011). A novel approach of image fusion on MR and CT images using wavelet transforms. 11:172-76.

G.Y. Wang, X.Z. Xu, X.Y. Jiang, R. Nie (2015). A modified model of pulse coupled neural networks with adaptive parameters and its application on image fusion. 6(9):2523-30.

Gautam, S., \& Kumar, M.(2015).An effective image fusion technique based on multi-resolution singular value decomposition. 14(2): 31-43.

H. Zhao, S.F. Ding (2014). Study of automated PCNN system based on fruit fly optimization algorithm. 10(15):6635-42.

Kakarala, R., \&togunbona, P.O. (2001). Signal analysis using multiresolution form of the singular value decomposition. 10(5): 724-735.

Kaur, A., \& Sharma, R (2016). Review on image fusion algorithm. 17(1):147-53.

Malini, S., AtMoni, R. S. (2015). Image denoising using multi-resolution singular value decomposition transform. 46:1708-15.

Naidu, V. P. S. (2011). Image fusion technique using multi-resolution singular value decomposition. 61(5): 479-84.

P.J. Burt, E.H. Adelson (1983). The Laplacian pyramid as a compact image code. 31 (4):532-40

S.Vasuki, S. Gandhimathi, S. Manika V Inodhini (2012). Comparative Analysis of Wavelets for Fusion Application. 12:40-44.

Shaveta, M., \&Arpinder .S (2014). A comparative analysis of different image fusion techniques. 2(1):815

Shih-Gu Huang(2010). Wavelet for Image Fusion. http : / / citeseerx . ist . psu edu/

Smt.G.Mamatha,L.Gayatri (2012). AN IMAGE FUSION USING WAVE-LET AND CURVELET TRANSFORMS. 1(2):2470-76. 
Subashini, M. M., EtSahoo, S. K.(2014).Pulse coupled neural networks and its applications. 41: 3965-74.

Varsha, M.,ct Kishore, M. R.(2014). Novel image fusion technique based on DWT \& MSVD. 2(8):2535-40.

Wang, Z.,Wang, S.,Zhu, Y.,AtMa, Y.(2015).Review of image fusion based on pulse-coupled neural network. 10:1-13.

X.Y. Jiang (2012). A self-adapting pulse-coupled neural network based on modified differential evolution algorithm and its application on image segmentation. 6(20):501-09.

X.Z. Xu, S.F. Ding, Z.P. Zhao, H. Zh (2011). Particle swarm optimization for automatic parameters determination of pulse coupled neural network. 6(8):1546-53.
X.Z. Xu, S.F. Ding, Z.Z. Shi, H. Zhu, Z.P. Zha (2012). A self-adaptive method for optimization the parameters of pulse coupled neural network based QPSO algorithm. 25(6):909-15.

Y. Li, X.J. Wu (2014). A novel image fusion method using self-adaptive dual-channel pulse coupled neural networks based on PSO evolutionary learning. 42(2):217-22.

Z. Wang, A.C. Bovik, H.R. Sheikh, et al., (2004). Image quality assessment: from error visibility to structural similarity. 13 (4):600-12.

Z.B. Wang, Y.D. Ma (2008). Medical image fusion using m-PCNN. 9(2):176-85. 